Maciej Bajorek*, Marek Kulczycki*, Marcin Ligas*

\title{
A Comparison of Iterative Methods of the Cubic Rate Convergence in the Problem of Transformation between Cartesian and Geodetic Coordinates**
}

\section{Introduction}

In geodetic practice there is often a need to transform from Cartesian to geodetic coordinates. Position of a point in Cartesian system may easily be expressed by geodetic coordinates as follows:

$$
\begin{gathered}
x=(N+h) \cos \varphi \cos \lambda \\
y=(N+h) \cos \varphi \sin \lambda \\
z=\left[N\left(1-e^{2}\right)+h\right] \sin \varphi
\end{gathered}
$$

where:

$$
\begin{aligned}
& \varphi- \text { geodetic latitude, } \\
& \lambda- \text { longitude, } \\
& h- \text { ellipsoidal height, } \\
& N=\frac{a}{\sqrt{1-e^{2} \sin ^{2} \varphi}}-\text { radius of curvature in the prime vertical, } \\
& e^{2}=\frac{a^{2}-b^{2}}{a^{2}}-\text { first eccentricity squared, } \\
& a-\text { length of the semimajor axis of an ellipsoid, } \\
& b-\text { length of the semiminor axis of an ellipsoid. }
\end{aligned}
$$

The inverse transform poses a more serious problem because of the necessity of solving a nonlinear equation. This task has been undertaken for the last decades and has

* AGH University of Science and Technology, Faculty of Mining Surveying and Environmental Engineering, Department of Geomatics, Krakow, Poland

** This paper is the result of research carried out within statutory research grant no. 11.11.150.006 in the Department of Geomatics, AGH University of Science and Technology, Krakow 
resulted in methods which may be classified into two groups: exact methods and iterative methods. The exact methods are usually based on the solution of a quartic equation with respect to an auxiliary variable leading to the solution e.g. $[1,7,18]$. The iterative methods rely on applying certain functions which iterate a solution with any accuracy e.g. $[8,13]$. Among the iterative methods there are rapidly convergent ones that achieve satisfying accuracy after only one iteration $[2,4]$. Both approaches have their virtues and drawbacks. Quartic equations lead to the exact solution in a finite number of steps $[9,17]$; allow for the solvability analysis [5] but engage many time consuming mathematical operations (square roots, trigonometric functions, hyperbolic functions). Iterative methods may be constructed in such a way to avoid time consuming mathematical operations but on the other hand they require many numerical tests confirming their convergence to the correct solution. Currently in the literature one can see the dominance of iterative solutions and one of them by Fukushima [4] is considered optimal.

\section{2. "Latitude Equation" and Its Solutions}

One possible solution to the problem of transformation is the use of "latitude equation" (e.g. Fukushima in [4]). Although the equation may be represented in any parameterization (geodetic, geocentric or parametric latitude) [12] in this study only parametric latitude will be used due to the analytical simplicity of the resulting equation. "Latitude equation" in this form may be written as:

$$
\left(a^{2}-b^{2}\right) \sin \psi \cos \psi-p a \sin \psi+z b \cos \psi=0
$$

where:

$\psi$ - parametric (reduced) latitude,

$p=\sqrt{x^{2}+y^{2}}$.

The solution to the above equation with respect to the parametric latitude $\psi$ gives the opportunity of finding unknown geodetic coordinates $(\varphi, h)$ on the basis of the following formulas:

$$
\left\{\begin{array}{l}
\varphi=\arctan \left(\frac{\tan \psi}{\sqrt{1-e^{2}}}\right) \\
h=\frac{p \sqrt{1-e^{2}} \cos \psi+z \sin \psi-b}{\sqrt{1-e^{2} \cos ^{2} \psi}}
\end{array}\right.
$$

It is easy to notice that equation (2) and the final formulas for conversion between Cartesian and geodetic coordinates (3) are loaded with a considerable amount of trigonometric functions. In problems where the time of execution of the whole numerical procedure is important the use of them is avoided due to the relatively long time of processing by computing machines. 
In order to limit their use in the coordinates transformation formulas Fukushima $[3,4]$ uses the substitution:

$$
t_{\psi}=\tan \psi, \cos \psi=\frac{1}{\sqrt{1+t_{\psi}^{2}}}, \sin \psi=\frac{t_{\psi}}{\sqrt{1+t_{\psi}{ }^{2}}}
$$

In this way "latitude equation" as a function of a tangent of the parametric latitude in an irrational form is obtained:

$$
f\left(t_{\psi}\right)=\frac{e^{2} t_{\psi}}{\sqrt{1+t_{\psi}^{2}}}-\frac{p}{a} t_{\psi}+\frac{b z}{a^{2}}=0
$$

The first and the second derivative of the function (5) necessary in iterative formulas of the third order convergence being the subject of this study are as follows:

$$
\begin{gathered}
f^{\prime}\left(t_{\psi}\right)=\frac{e^{2}}{{\sqrt{t_{\psi}^{2}+1}}^{3}}-\frac{p}{a} \\
f^{\prime \prime}\left(t_{\psi}\right)=\frac{-3 e^{2} t_{\psi}}{{\sqrt{t_{\psi}^{2}+1}}^{5}}
\end{gathered}
$$

The initial value of the iterative process has been adopted as a tangent of the parametric latitude for the zero geodetic (ellipsoidal) height i.e.:

$$
t_{\psi}^{0}=\frac{a z}{b p}=\frac{z}{\sqrt{1-e^{2}} p}
$$

The final values of the geodetic latitude and height in this case are expressed as a (modification of the formula (3)):

$$
\left\{\begin{array}{l}
\varphi=\arctan \left(\frac{t_{\psi}}{\sqrt{1-e^{2}}}\right) \\
h=\frac{p \sqrt{1-e^{2}}+z t_{\psi}-b \sqrt{1+t_{\psi}^{2}}}{\sqrt{1-e^{2}+t_{\psi}^{2}}}
\end{array}\right.
$$

By simple manipulations the irrational equation (5) may be presented as a quartic equation of the form:

$$
f\left(t_{\psi}\right)=t_{\psi}{ }^{4}-2 K t_{\psi}{ }^{3}+\left(1+K^{2}-L^{2}\right) t_{\psi}{ }^{2}-2 K t_{\psi}+K^{2}=0
$$

and the first and second derivative take the following form:

$$
f^{\prime}\left(t_{\psi}\right)=4 t_{\psi}{ }^{3}-6 K t_{\psi}{ }^{2}+2\left(1+K^{2}-L^{2}\right) t_{\psi}-2 K
$$


and:

$$
f^{\prime \prime}\left(t_{\psi}\right)=12 t_{\psi}^{2}-12 K t_{\psi}+2\left(1+K^{2}-L^{2}\right)
$$

where:

$$
K=\frac{b z}{a p}=\frac{z \sqrt{1-e^{2}}}{p}, \quad L^{2}=\frac{e^{4} a^{2}}{p^{2}} .
$$

According to Abel-Ruffini's theorem quartic equations are the last which can be solved through radicals. Despite this, iterative approach is more often applied due to the complexity of achieving the solution in a strict approach (complex solutions, trigonometric and hyperbolic functions, intermediate solution of the cubic equation etc.). Applying Euler-Weierstrass substitution (often used in integral calculus) to the initial latitude equation (2), i.e.:

$$
T=\tan \frac{1}{2} \psi, \cos \psi=\frac{1-T^{2}}{1+T^{2}}, \sin \psi=\frac{2 T}{1+T^{2}}
$$

one obtains a quartic equation in a simple form:

$$
f(T)=T^{4}+2 E T^{3}+2 F T-1=0
$$

and the first and second derivative read:

$$
\begin{gathered}
f^{\prime}(T)=4 T^{3}+6 E T^{2}+2 F \\
f^{\prime \prime}(T)=12 T^{2}+12 E T
\end{gathered}
$$

where:

$$
E=\frac{\left(a^{2}-b^{2}+a p\right)}{b z}, F=\frac{a p+b^{2}-a^{2}}{b z} .
$$

Initial value of the iterative process has been adopted as a tangent of half of the parametric latitude for the zero geodetic height i.e.:

$$
T^{0}=\frac{z}{p \sqrt{1-e^{2}}+\sqrt{\left(1-e^{2}\right) p^{2}+z^{2}}}
$$

The final values of the geodetic latitude and height in this case are expressed as:

$$
\left\{\begin{array}{l}
\varphi=\arctan \left(\frac{2 T}{\sqrt{1-e^{2}}\left(1-T^{2}\right)}\right) \\
h=\frac{p \sqrt{1-e^{2}}\left(1-T^{2}\right)+2 z T-b\left(1+T^{2}\right)}{\sqrt{\left(1+T^{2}\right)^{2}-e^{2}\left(1-T^{2}\right)^{2}}}
\end{array}\right.
$$


Applying "inverted" with respect to (13) substitution (replacement in the sine and cosine functions) to the equation (2) one obtains:

$$
T=\tan \left(\frac{\pi}{4}-\frac{\psi}{2}\right), \sin \psi=\frac{1-T^{2}}{1+T^{2}}, \cos \psi=\frac{2 T}{1+T^{2}}
$$

From this substitution the following quartic equation is obtained:

$$
f(T)=T^{4}+2 G T^{3}+2 H T-1=0
$$

And simple expressions for the first and second derivative take the form:

$$
\begin{gathered}
f^{\prime}(T)=4 T^{3}+6 G T^{2}+2 H \\
f^{\prime \prime}(T)=12 T^{2}+12 G T
\end{gathered}
$$

where:

$$
G=\frac{\left[z b-\left(a^{2}-b^{2}\right)\right]}{a p}, H=\frac{\left[z b+\left(a^{2}-b^{2}\right)\right]}{a p} .
$$

The initial value for the iterative process (for $h=0$ ) reads:

$$
T^{0}=\frac{p \sqrt{1-e^{2}}+\sqrt{\left(1-e^{2}\right) p^{2}+z^{2}}-z}{p \sqrt{1-e^{2}}+\sqrt{\left(1-e^{2}\right) p^{2}+z^{2}}+z}
$$

The final values of the geodetic latitude and height in this case are as follows:

$$
\left\{\begin{array}{l}
\varphi=\arctan \left(\frac{1-T^{2}}{2 \sqrt{1-e^{2}} T}\right) \\
h=\frac{2 p \sqrt{1-e^{2}} T+z\left(1-T^{2}\right)-b\left(1+T^{2}\right)}{\sqrt{\left(1+T^{2}\right)^{2}-4 e^{2} T^{2}}}
\end{array}\right.
$$

\section{Some remarks}

Irrational equation (5) differs somewhat from the original equation presented by Fukushima [4]. Fukushima introduces the absolute value of the $z$ Cartesian coordinate to the equation. Due to the symmetry of the ellipsoid of revolution, its section with a plane containing the $z$ axis (polar) is always an identical meridian ellipse thus Fukushima solves the problem within the interval of latitudes $\left(0-90^{\circ}\right)$. The appropriate sign of the latitude is restored in relation to the sign of the $z$ Cartesian 
coordinate. Equation (20) is nothing but famous Borkowski's equation - one of the first quartic equations in the geodetic literature concerning the problem of conversion. Borkowski [1] solves this equation in a strict way using Ferrari's method. The derivation of Borkowski's equation presented here differs slightly from the original. Borkowski [1] begins with "latitude equation" in the geodetic latitude parameterization and uses the substitution:

$$
\tan \varphi=\frac{a\left(1-t^{2}\right)}{2 b t}
$$

In this study it is visible that starting with "latitude equation" in the parametric latitude parameterization simplifies the derivation of computational formulas considerably. Equation (14) has a singularity for $z=0$ (equator), equation (20) has a singularity for $p=0$, both cases are easy to eliminate with a simple "if" statement. While in the geodetic literature it is a common belief that the strict solution to the problem of transformation by solving quartic equation reaches the 70 's of the last century, in the work dedicated to conic sections by Sommerville ([15], first published in 1924) one can find the "latitude equation" in the parameterization of the reduced latitude together with the substitution (13) giving a quartic equation.

\section{Cubic Rate Convergence Iterative Methods for Solving Nonlinear Equations}

In order to solve the latitude equation transformed to the irrational form (5) and polynomial form (10) and (14) five iterative methods of the cubic rate convergence have been used. The four among them belong to the so called Tschebyscheff-Halley family of iterative methods and these are: Halley's, super-Halley's, Tschebyscheff's and Cauchy's methods. In addition, a method dedicated to solving polynomial equations - Laguerre's method has been used. Below there are listed iterative formulas for each method:

- Halley's method:

$$
t_{i+1}=t_{i}-\frac{2 f\left(t_{i}\right) f^{\prime}\left(t_{i}\right)}{2\left[f^{\prime}\left(t_{i}\right)\right]^{2}-f\left(t_{i}\right) f^{\prime \prime}\left(t_{i}\right)}
$$

- super-Halley's method:

$$
t_{i+1}=t_{i}-\frac{1}{2}\left(\frac{f\left(t_{i}\right)}{f^{\prime}\left(t_{i}\right)}+\frac{f\left(t_{i}\right) f^{\prime}\left(t_{i}\right)}{\left[f^{\prime}\left(t_{i}\right)\right]^{2}-f\left(t_{i}\right) f^{\prime \prime}\left(t_{i}\right)}\right)
$$


- Tschebyscheff's method:

$$
t_{i+1}=t_{i}-\left(1+\frac{f\left(t_{i}\right) f^{\prime \prime}\left(t_{i}\right)}{2\left[f^{\prime}\left(t_{i}\right)\right]^{2}}\right) \frac{f\left(t_{i}\right)}{f^{\prime}\left(t_{i}\right)}
$$

- Cauchy's method:

- Laguerre's method:

$$
t_{i+1}=t_{i}-\frac{2}{1+\sqrt{1-\frac{2 f^{\prime \prime}\left(t_{i}\right) f\left(t_{i}\right)}{\left[f^{\prime}\left(t_{i}\right)\right]^{2}}}} \frac{f\left(t_{i}\right)}{f^{\prime}\left(t_{i}\right)}
$$

$$
\begin{gathered}
t_{i+1}=t_{i}-\frac{n f\left(t_{i}\right)}{f^{\prime}\left(t_{i}\right) \pm \sqrt{H\left(t_{i}\right)}} \\
H\left(t_{i}\right)=(n-1)\left\{(n-1)\left[f^{\prime}\left(t_{i}\right)\right]^{2}-n f\left(t_{i}\right) f^{\prime \prime}\left(t_{i}\right)\right\}
\end{gathered}
$$

where:

$$
\begin{aligned}
i & - \text { iteration's number, } \\
f(\ldots), f^{\prime}(\ldots) f^{\prime \prime}(\ldots) & -\quad \text { function resulting from an appropriate equation, its first } \\
& \text { and second derivative, } \\
n- & \text { polynomial's degree. }
\end{aligned}
$$

The sign in the denominator of (30) is chosen to give the larger absolute value.

Derivations of the methods as well as their convergence criteria interested reader may find in e.g. [6, 10, 11, 16, 17].

\section{Tests and Results}

Tests of the five iterative methods for the three representations of "latitude equation" (5), (10) and (14) were conducted for two height intervals and 0-90(step of $0.05^{\circ}$ ) interval of the geodetic latitude (quadrant of a meridian ellipse, $z \geq 0$ ). The first height interval (case A) covered ellipsoidal heights from $-10 \mathrm{~km}$ to $10 \mathrm{~km}$ with a step of $50 \mathrm{~m}$ and the second one (case B) covered heights from $10 \mathrm{~km}$ to $36000 \mathrm{~km}$ (altitude of geostationary satellites) with a step of $25 \mathrm{~km}$. All the algorithms have been coded in Borland Delphi environment. Source codes are available from the authors upon request. All constant expressions (e.g. $\left.\sqrt{1-e^{2}}, e^{4} a^{2}\right)$ have been declared once at the beginning of a driver program and had no impact on the execution time of any numerical procedure. Parameters of the reference ellipsoid GRS80 were adopted from [14]. In case of polynomial representation Horner's scheme was used in order to minimize multiplication operations form $n(n+1) / 2$ (classical representation) 
to $n$ (where $n$ stands for polynomial's degree). In the first step the driver program generated Cartesian coordinates $(x, y, z)$ on the basis of theoretical geodetic coordinates $(\varphi, \lambda, h)$. In the second step Cartesian coordinates were converted into geodetic ones and compared with their theoretical values from the first step. In order to asses a transformation error of each method quantities $E_{-} h$ and $E \_\varphi$ were introduced. They are the maximum differences in absolute value between theoretical values and those recomputed. Besides the transformation errors the time of execution of each numerical procedure was measured and finally standardized to the time of execution of numerical procedure solving the irrational equation with Halley's method. Results for the two cases (A and B) are listed in Tables 1-3. For the case A (from -10 $\mathrm{km}$ to $10 \mathrm{~km}$ ) the number of iterations was limited to one for each method, for the case B maximum of four iterations was performed depending on the accuracy of the backward transformation. Errors for the ellipsoidal height less than $10^{-2} \mathrm{~mm}$ were omitted as well as errors for the geodetic latitude less than $10^{-5}$ arcsecond (").

Table 1. Standardized times (ST) of execution of numerical procedures, maximum error for ellipsoidal height $\left(E \_h\right)[\mathrm{mm}]$, maximum error for geodetic latitude $\left(E \_\varphi\right)$ ["]

\begin{tabular}{||l|c|c|c|c|c|c|c|c|c||}
\hline \multicolumn{10}{|c|}{ Case A - one iteration } \\
\cline { 2 - 12 } & \multicolumn{3}{|c|}{ Equation (5) } & \multicolumn{3}{c||}{ Equation (10) } & \multicolumn{3}{c||}{ Equation (14) } \\
\cline { 2 - 11 } & $S T$ & $\begin{array}{c}E \_h \\
{[\mathrm{~mm}]}\end{array}$ & $\begin{array}{c}E_{-} \varphi \\
{["]}\end{array}$ & $S T$ & $\begin{array}{c}E \_h \\
{[\mathrm{~mm}]}\end{array}$ & $\begin{array}{c}\text { E_ } \varphi \\
{["]}\end{array}$ & ST & $\begin{array}{c}\text { E_h } \\
{[\mathrm{mm}]}\end{array}$ & $\begin{array}{c}\text { E_ } \varphi \\
{["]}\end{array}$ \\
\hline Halley's method & 1.00 & - & - & 1.02 & - & - & 1.06 & - & - \\
\hline Super-Halley's method & 0.99 & - & - & 1.00 & - & - & 1.09 & - & - \\
\hline Tschebyscheff's method & 1.03 & - & - & 1.02 & - & - & 1.15 & - & - \\
\hline Cauchy's method & 1.17 & - & - & 1.15 & - & - & 1.17 & - & - \\
\hline Laguerre's method & N/A & N/A & N/A & 1.07 & - & - & 1.17 & - & - \\
\hline
\end{tabular}

${ }^{*} \mathrm{~N} / \mathrm{A}$ - not applicable

Table 2. Standardized times (ST) of execution of numerical procedures, maximum error for ellipsoidal height $\left(E_{-} h\right)[\mathrm{mm}]$, maximum error for geodetic latitude $\left(E_{-} \varphi\right)\left[{ }^{\prime \prime}\right]$

\begin{tabular}{|c|c|c|c|c|c|c|c|c|c|}
\hline \multicolumn{10}{|c|}{ Case B - one iteration } \\
\hline \multirow[b]{2}{*}{ Method } & \multicolumn{3}{|c|}{ Equation (5) } & \multicolumn{3}{|c|}{ Equation (10) } & \multicolumn{3}{|c|}{ Equation (14) } \\
\hline & ST & $\begin{array}{c}E \_h \\
{[\mathrm{~mm}]}\end{array}$ & $\begin{array}{c}E \_\varphi \\
{["]}\end{array}$ & ST & $\begin{array}{c}E \_h \\
{[\mathrm{~mm}]}\end{array}$ & $\begin{array}{c}E_{-} \varphi \\
{["]}\end{array}$ & ST & $\begin{array}{c}E \_h \\
{[\mathrm{~mm}]}\end{array}$ & $\begin{array}{c}E \_\varphi \\
{["]}\end{array}$ \\
\hline Halley's method & 1.00 & - & - & 1.02 & 9958 & 141 & 1.05 & - & 0.0008 \\
\hline Super-Halley's method & 0.99 & - & - & 0.99 & 3858 & 88 & 1.09 & - & 0.002 \\
\hline Tschebyscheff's method & 1.03 & - & - & 1.02 & 14141 & 169 & 1.15 & - & 0.003 \\
\hline Cauchy's method & 1.17 & - & - & 1.14 & 111 & 15 & 1.17 & - & 0.002 \\
\hline Laguerre's method & N/A & N/A & N/A & 1.07 & 4956 & 100 & 1.17 & - & 0.001 \\
\hline
\end{tabular}

${ }^{*} \mathrm{~N} / \mathrm{A}$ - not applicable 
Table 3. Standardized times (ST) of execution of numerical procedures, maximum error for ellipsoidal height $\left(E \_h\right)[\mathrm{mm}]$, maximum error for geodetic latitude $\left(E_{-} \varphi\right)$ ["]

\begin{tabular}{|c|c|c|c|c|c|c|c|c|c|}
\hline \multicolumn{10}{|c|}{ Case B - two iterations } \\
\hline \multirow[b]{2}{*}{ Method } & \multicolumn{3}{|c|}{ Equation (5) } & \multicolumn{3}{|c|}{ Equation (10) } & \multicolumn{3}{|c|}{ Equation (14) } \\
\hline & ST & $\begin{array}{c}E_{-} h \\
{[\mathrm{~mm}]}\end{array}$ & $\begin{array}{c}E_{-} \varphi \\
{["]}\end{array}$ & ST & $\begin{array}{c}E \_h \\
{[\mathrm{~mm}]}\end{array}$ & $\begin{array}{c}E \_\varphi \\
{["]}\end{array}$ & ST & $\begin{array}{c}E \_h \\
{[\mathrm{~mm}]}\end{array}$ & $\begin{array}{c}E \_\varphi \\
{["]}\end{array}$ \\
\hline Halley's method & 1.00 & - & - & 0.95 & 109 & 15 & 0.99 & - & - \\
\hline Super-Halley's method & 1.03 & - & - & 0.98 & 1 & 2 & 1.03 & - & - \\
\hline Tschebyscheff's method & 1.09 & - & - & 1.02 & 372 & 27 & 1.06 & - & - \\
\hline Cauchy's method & 1.22 & - & - & 1.19 & - & 0.0005 & 1.23 & - & - \\
\hline Laguerre's method & N/A & N/A & N/A & 1.11 & 7 & 4 & 1.15 & - & - \\
\hline
\end{tabular}

${ }^{*} \mathrm{~N} / \mathrm{A}$ - not applicable

Results presented in Table 1 reveal that for the case A (from $-10 \mathrm{~km}$ to $10 \mathrm{~km}$ ) all representations of "latitude equation" as well as all applied methods assure errors of less than $10^{-2} \mathrm{~mm}$ for the ellipsoidal height and less than $10^{-5}$ " for the geodetic latitude after only one iteration. For the first three iterative methods standardized timings are comparable. The exception is the equation (14) solved by Tschebyscheff's method where a significant difference is visible. Significant differences in timings are also visible for the three equations solved by Cauchy's method and for two polynomial representations with the use of Laguerre's method. In the case of the last two mentioned longer time of execution is caused by an additional square root operation included in the iteration functions. Results contained in Table 2 (one iteration) and Table 3 (two iterations) concern the case B (from $10 \mathrm{~km}$ to $36000 \mathrm{~km}$ ). From Table 2 one notices immediately that the irrational representation of the "latitude equation" assures high accuracy of the transformation after only one iteration independent of the numerical method of solving. Also polynomial form (14) gives quite accurate results after one iteration, for the ellipsoidal height satisfactory and for the geodetic latitude on the level of $\pm 3 \cdot 10^{-3}$ ". The second iteration, in this case, assures more than satisfactory results. Polynomial representation (10) requires 3-4 iterations to reach assumed level of accuracy in the coordinates' conversion. As seen, the most universal (range of heights and time of execution) is the irrational form of the "latitude equation" solved by Halley's, super-Halley's and Tschebyscheff's methods. Differences in timings for these three methods are so slight that they could be influenced by the unequal work of CPU and other processes running in the background while performing the test. These results support the intuition and experience of Fukushima in the selection of this particular representation of "latitude equation" and Halley's method to solve the problem of transformation from Cartesian coordinates to geodetic ones. 


\section{Conclusions}

A comparison of efficiency of five iterative methods (Halley, super-Halley, Tschebyscheff, Cauchy and Laguerre) with a cubic rate convergence has been presented. The iterative methods have been applied to the problem of conversion between Cartesian and geodetic coordinates. The mentioned methods have been applied to the solution of the "latitude equation" in three different representations - one of them is an irrational form and the others are in a polynomial form. Among the tested numerical methods of solving nonlinear equations three methods; namely: Halley's, super-Halley's and Tschebyscheff's methods behaved visibly better than the other two. Also, among three representations of the "latitude equation" the irrational form assures high accuracy of the transformation with the lowest computational cost in comparison to the remaining two representations. The polynomial representation in the form of (14) is slower by several percents but almost equally accurate. The polynomial representation in the form of (10) could compete with the abovementioned only for the small range of ellipsoidal heights because it loses accuracy considerably with increasing heights thus it lacks signs of universality. In conclusion, it must be noted that the results obtained in this study cannot be regarded as certain in any case because they are dependent on the programming environment and the computing platform on which numerical tests are performed.

\section{References}

[1] Borkowski K.: Transformation of geocentric to geodetic coordinates without approximation. Astrophysics and Space Science, vol. 139, 1987, pp. 1-4.

[2] Bowring B.R.: Transformation from spatial to geographical coordinates. Survey Review, vol. 23, 1976, pp. 323-327.

[3] Fukushima T.: Fast transform from geocentric to geodetic coordinates. Journal of Geodesy, vol. 73(11), 1999, pp. 603-610.

[4] Fukushima T.: Transformation from Cartesian to geodetic coordinates accelerated by Halley's method. Journal of Geodesy, vol. 79(12), 2006, pp. 689-693.

[5] Gonzalez-Vega L., Polo-Blanco I.: A symbolic analysis of Vermeille and Borkowski polynomials for transforming $3 D$ artesian to geodetic coordinates. Journal of Geodesy, vol. 83(11), 2009, pp. 1071-1081.

[6] Gutierrez J.M., Hernandez M.A.: An acceleration of Newton's method: SuperHalley method. Applied Mathematics and Computation, vol. 117(2-3), 2001, pp. 223-239.

[7] Hedgley D.R., Edwards Flight Research Center (Calif): An exact transformation from geocentric to geodetic coordinates for nonzero altitudes. NASA Technical Report 458, United States National Aeronautics and Space Administration, Washington 1976. 
[8] Heiskanen W.A., Moritz H.: Physical Geodesy. W.H. Freeman and Company, San Francisco 1967.

[9] Hobson E.W.: A treatise on plane trigonometry. Cambridge at the University Press 1918.

[10] Kincaid D., Cheney W.: Numerical Analysis. Brooks / Cole Publishing Company, Pacific Grove, California, 1991.

[11] Kou J.: Some variants of Cauchy's method with accelerated fourth-order convergence. Journal of Computational and Applied Mathematics, vol. 213(1), 2008, pp. 71-78.

[12] Ligas M.: Various parameterizations of "latitude" equation - Cartesian to geodetic coordinates transformation. Journal of Geodetic Science, vol. 3(2), 2013, pp. 87-94.

[13] Lin K.C., Wang J.: Transformation from geocentric to geodetic coordinates using Newton's iteration. Bulletin Geodesique, vol. 69, 1995, pp. 300-303.

[14] Moritz H.: Geodetic Reference System 1980. Bulletin Geodesique, vol. 54, 1980, pp. 395-405.

[15] Sommerville D.M.Y.: Analytical conics. G. Bell and sons, London 1961.

[16] Wang H.: On new third-order convergent iterative formulas. Numerical Algorithms, vol. 48(4), 2008, pp. 317-325.

[17] Zaguskin W.L.: Przeglad metod numerycznych rozwiazywania równań. PWN, Warszawa 1965.

[18] Vermeille H.: Direct transformation from geocentric coordinates to geodetic coordinates. Journal of Geodesy, vol. 76(8), 2002, pp. 451-454. 